\title{
Estudio Clínico y Enfoque Terapéutico de la Incontinencia Urinaria en la Mujer
}

\author{
Dres.: Hernán Redondo Gómez*, Raúl Cruz Palacio**, \\ Alfonso López Jiménez*** Carlos Hernández Forero****.
}

\section{INTRODUCCION}

La Incontinencia Urinaria (I.U.) ha existido desde tiempo inmemorial $y$ sigue siendo un problema sin solución adecuada en nuestros días. Lo atestigua la literatura que día a día se publica para plantear nuevos métodos de estudio y tratamiento $(1,2,3,17,18,21,24)$. El conocimiento actual de la fisiología normal y la fisiopatología de la I.U. obliga a realizar un estudio adecuado antes de afrontar el tratamiento.(3, $15,16,24,25)$.

* Ginecólogo Obstetra, Hospital Regional Simón Bolívar. Instructor, Escuela Colombiana de Medicina.

** Urólogo, Hospital Regional Simón Bolívar. Instructor, Escuela Colombiana de Medicina.

*** Ginecólogo Obstetra, Hospital Regional Simón Bolívar. Instructor, Escuela Colombiana de Medicina.

**** Urólogo, Fellow en Urodinamia Clínica. Colaboración especial.
Salvo contadas excepciones, ya no se considera la operación de Kelly como la indicada para corregir la incontinencia urinaria de esfuerzo (I.U.E.). La bibliografía revisada señala amplia posibilidad de fracaso entre el $35 \%$ y el $52 \%$ (6, $8,9,10,14,21,22,24,26)$. Por esta razón en los últimos años han surgido otras técnicas con mejores posibilidades, como las de Burch, Tanagho, Powell, Stamey y Pereyra $(2,17,20,21,23$, 27). No nos inclinamos por una técnica en particular para todos los casos. Más bien la escogemos según conveniencia individual, de acuerdo con criterios clínicos bien definidos, para obtener mejor rendimiento.

Desafortunadamente no disponemos de cifras exactas de la I.U. en nuestro medio. Sin embargo, sabemos que es motivo de consulta muy frecuente asociado a otros trastornos como leucorreas, infección urinaria y dispareunia. Además, la I.U. femenina aumenta progresivamente al aparecer por factores de riesgo como edad, multiparidad, obesidad $y$ prolapso genital. 
Pretendemos con este trabajo aportar un concepto multidisciplinario a la solución de este trastorno en la mujer.

\section{Objetivos generales:}

1. Proponer un protocolo de estudio multidisciplinario de la I.U. en la mujer.

2. Proponer un enfoque terapéutico de la I.U. de la mujer de nuestro medio, con nuestros recursos.

\section{Objetivos específicos:}

1. Realizar un trabajo prospectivo a dos, cinco y diez años de seguimiento a partir de agosto de 1983, con las pacientes de la Clínica de Incontinencia Urinaria del Hospital Simón Bolívar de Bogotá, Regional del Distrito Especial y área de influencia de la Escuela Colombiana de Medicina.

Presentamos en este informe los resultados preliminares de dos años.

\section{Definición de Conceptos:}

Consideramos de suma importancia precisar términos y conceptos empleados antes de presentar los resultados.

Incontinencia Urinaria (I.U.): Tomando la definición de $\mathrm{C}$. Kaser, se entiende por I.U. como la emisión involuntaria de orina por la región genital (11). Como se observa, es este un concepto muy amplio, por lo cual es preciso clasificar la I.U. de acuerdo con su etiología y de acuerdo al grado de severidad.

Clasificación de la I.U. de acuerdo con la etiología: Modificada de Gallo (7).

\section{I. - I.U. intermitente}
a - I.U.E.
b - I. de urgencia.

c - I.U. paradójica, por sobredistensión o rebosamiento.

d - Enuresis del adulto.

e - I.U. por inestabilidad vesical o funcional.

\section{$I I-I . U$. continua}
a. - I.U. real o neurogénica (esfinter fláscido).
b - Fistulas - Congénitas
- Adquiridas.

Clasificación de la I.U. de acuerdo con la severidad:

GRADO I: Se presenta sólo con los grandes esfuerzos, es ocasional (una o dos veces al mes), generalmente en posición vertical

GRADO II: Se presenta con esfuerzos moderados, es frecuente (una o dos veces por semana), con cambios bruscos de posición.

GRADO III: Se presenta con pequeños esfuerzos, es muy frecuente (casi todos los días), en posición vertical, sentada e inclusive acostada y durante el coito.

I.U.E.: Llamada también anatómica, es la pérdida involuntaria de orina por la uretra íntegra con los aumentos de la presión intra-abdominal, en presencia de vejiga estable. (Modificada de Ullery (29)).

Vejiga Inestable (V.I.): Se presenta cuando aparecen contracciones no inhibidas del músculo detrusor superiores a $15 \mathrm{~cm}$ de agua, durante la fase de llenado vesical, por falta de coordinación entre el esfinter uretral externo $y$ el detrusor. El diagnóstico se sospecha clí. 
nicamente y se confirma con la cistometría de provocación (3) (Fig. 4).

\section{Estudio Urodinámico (E.U.): Com-} prende la uroflujometría, la electromiografia esfinteriana, la cistometria y el perfil de presión uretral. (Este último estudio no fue practicado).

Indicaciones establecidas en el presente trabajo para solicitar E.U.:

- Antecedente de cirugía para corregir I.U.E.

- Frecuencia, nicturia, urgencia e incontinencia de urgencia.

- Enuresis del adulto.

- Falla en el control voluntario de la micción.

- Nulípara con I.U.

- Pacientes muy seniles.
- Pacientes siquiátricas.

- Pacientes diabéticas.

- Capacidad vesical inferior a 300 cc o superior a $800 \mathrm{cc}$.

Uroflujometría.: Es el estudio del flujo urinario en términos de volúmen sobre tiempo (cc $x$ seg.) a través de la uretra durante la fase de micción. Se analiza el flujo urinario promedio, el máximo flujo urinario y el trazado (Fig. 1).

Electromiografía esfinteriana: Es el registro del potencial eléctrico producido por la actividad muscular estriada del esfinter uretral externo, aplicando electrodos parauretrales, tanto en fase de llenado como de micción (Fig. 2).

Cistometria: Es el estudio de los cambios de presión y la adaptabilidad (compliance) vesical con el aumento paulatino de volúmenes de agua en fase de llenado. (Figs. 3 y 4 ).

Figura No. 1

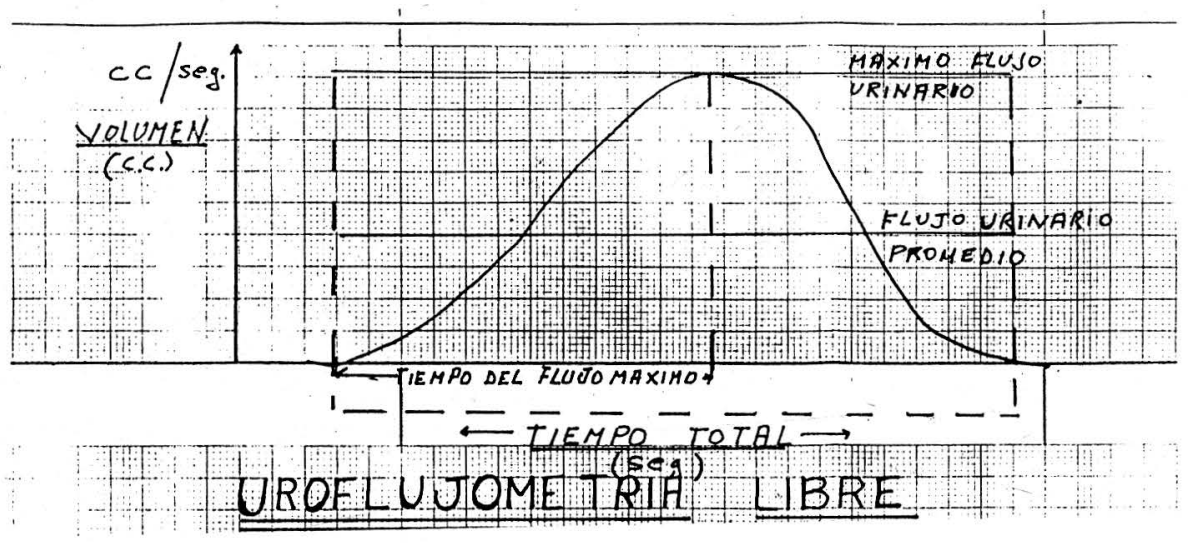


Figura No. 2

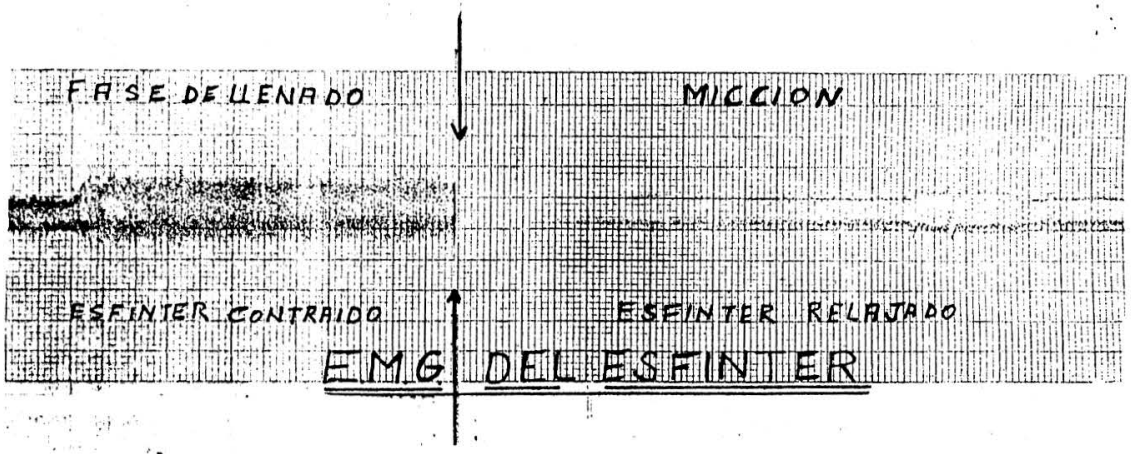

Figura No. 3
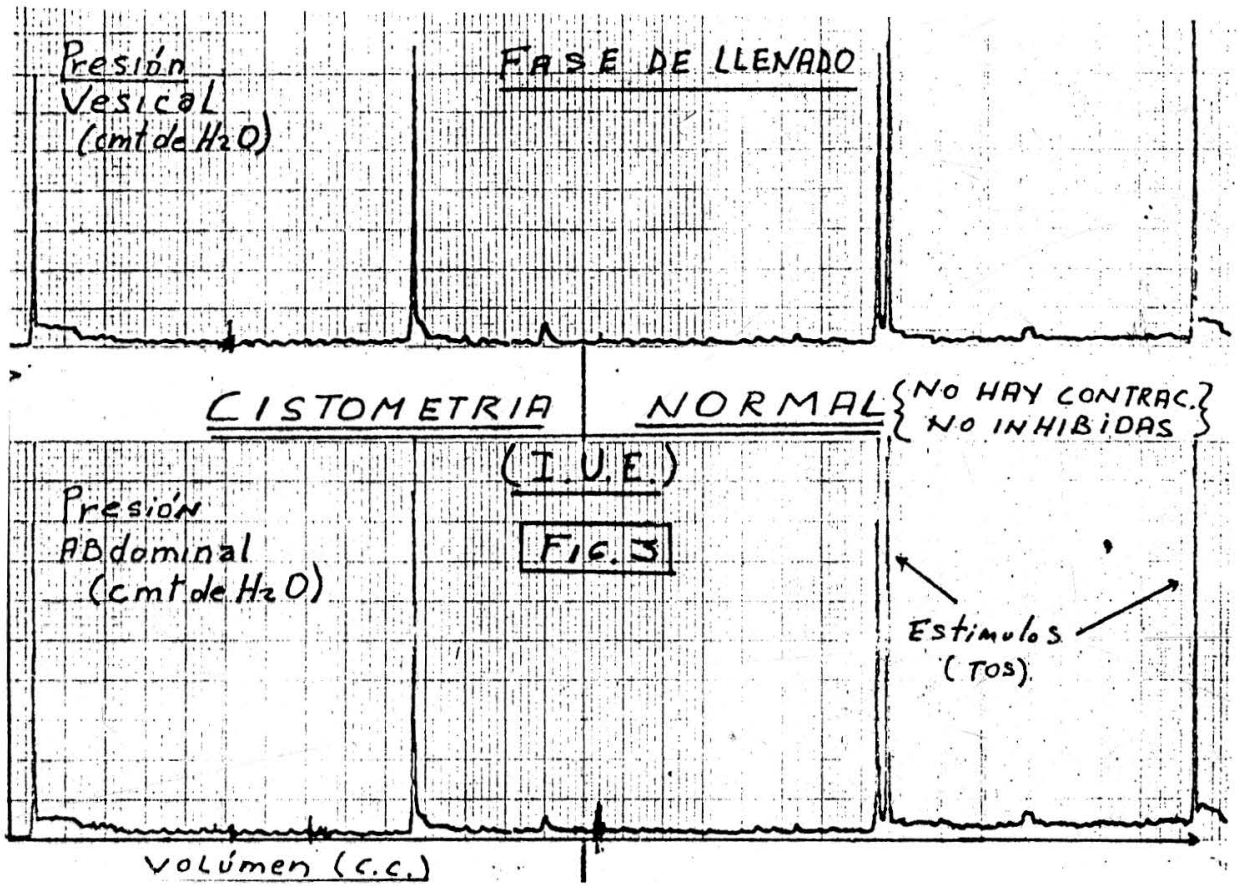
Figura No. 4

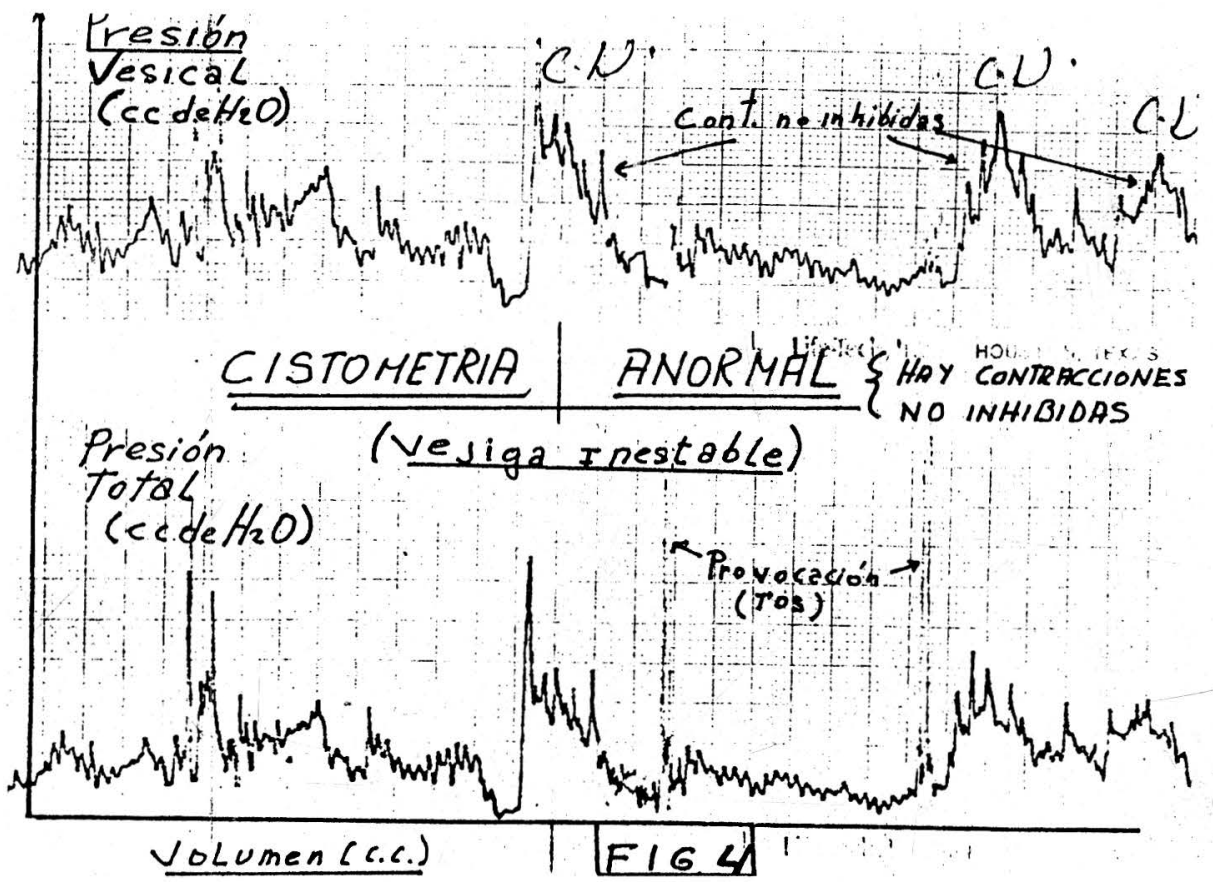

\section{METODOS}

Se realizó un estudio prospectivo de las pacientes de la Clínica de Incontinencia Urinaria del Hospital Regional Simón Bolívar de Bogotá, desde agosto de 1983 hasta el 31 de julio de 1985, con un total de 164 pacientes.

Se excluyeron del estudio las pacientes con I.U. grado I y los casos de infección urinaria activa a quienes se les dio tratamiento específico y luego se revaluaron.

Todas las pacientes fueron estudiadas de acuerdo al siguiente protocolo:
A. La paciente ingresa por consulta externa general, Urología o Ginecología, donde se elabora la historia clínica y se solicitan los exámenes de rutina, incluido el urocultivo.

B. Pasa a la Clínica de Incontinencia donde se realiza el interrogatorio específico, se descarta la infección urinaria y se confirma la presencia de I.U.

C. Se practica examen urológico y ginecológico completo, en forma simultánea.

D. Se practica medición, calibración y dilatación uretral si esta es necesaria. 
F. Se practica cistoscopia.

G. Se practica E.U., de acuerdo con las indicaciones anotadas.

H. Una vez establecido el diagnóstico del tipo de I.U., se distribuyeron en cuatro grupos terapéuticos de acuerdo con los siguientes criterios clínicos selectivos:

GRUPO I: (a) Operación de Tanagho simple o asociada a otra cirugía abdominal.

(b) Operación de Tanagho más perineorrafia.

Pacientes con I.U.E., vejiga estable, uretrocistocele grado | o $\|$ de poco componente posterior, preferiblemente delgadas, con o sin patología abdominal asociada como miomatosis uterina. (a) Sin rectocele o desgarro perineal (b) Con rectocele o desgarro perineal.

GRUPO II: Operación de Powell.

Pacientes con I.U.E., vejiga estable, excesivamente obesas o con cistocele grado III de gran componente posterior o con grados quirúrgicos de histerocele, cuello elongado, enterocele, o desgarro perineal III.

GRUPO III: Operación de Stamey Pereyra.

Recientemente iniciado, incluye pacientes con I.U.E., vejiga estable, obesas y con poca patología vaginal asociada.

GRUPO IV: Tratamiento Médico.

Pacientes con diagnóstico confirmado por E.U. de I.U. por inestabilidad vesical. Recibieron el siguiente tratamiento:

- Dilatación uretral.

- Ejercicios vesicales de Keegel.
- Fenilpropanolamina oral, dosis entre $50 \mathrm{mg}$ y $150 \mathrm{mg}$ al día.

- Bromuro de propantelina oral, 30 $\mathrm{mg}$ a $45 \mathrm{mg}$ al día.

- Antisépticos urinarios como Acido nalidíxico, Mandelato de metenamina y Nitrofurantoina a dosis habituales.

- Estrógenos conjugados locales en pacientes con signos de hipoestrogenismo vaginal.

\section{RESULTADOS}

Un total de 164 casos estudiados, los cuales se distribuyeron en los cuatro grupos terapéuticos así:

\section{GRUPO I}

Número total de operaciones de Tanagho realizadas $103,62.8 \%$ del total y $75 \%$ de los casos quirúrgicos.

a) Operación de Tanagho simple o asociada a otra cirugía abdominal 56 casos, $34.1 \%$ del total y $54.3 \%$ de los casos de I.U.E., distribuidos así:

- Operación de Tanagho simple, 46 casos.

- Operación de Tanagho más histerectomía abdominal, 8 casos.

- Operación de Tanagho más histerectomía abdominal ampliada, 1 caso.

- Operación de Tanagho más extirpación de quiste de ovario, 1 caso.

b) Operación de Tanagho más perineorrafia 47 casos, 28.6\% del total y $34.3 \%$ de los casos quirúrgicos.

\section{GRUPO II:}

Número total de Operaciones de Powell realizadas, 30 casos, $18.2 \%$ del 
total y 21.8\% de los casos de I.U.E., distribuidos así:

- Operación de Powell más colpoperineorrafia, 17 casos.

- Operación de Powell más histerectomía vaginal, 5 casos.

- Operación de Powell más operación de Manchester Fothergill y colpoperineorrafia, 5 casos.

- Operación de Powell más corrección de desgarro 111 complicado, 2 casos.

- Operación de Powell más corrección de enterocele III y colpoperineorrafia, 1 caso.

GRUPO III: (recientemente incluido)

Número de operaciones de Stamey Pereyra realizadas $4,2.4 \%$ del total y 2.9\% de los casos de I.U.E.

\section{GRUPO IV:}

Se encontró un total de 27 casos de vejiga inestable confirmados por urodinamia, $16.4 \%$ del total y 137 casos de I.U.E., $83.5 \%$

Se practicaron 61 estudios urodinámicos (37.1\% del total), de los cuales 34 correspondieron a vejigas estables (55.7\%) y 27 a inestables (44.2\%).

\section{Eơad}

La edad de las pacientes osciló entre 25 y 78 años. Reportamos estos datos por separado tanto de I.U.E. (tabla No. 1, Fig. 5) como de V.I. (tabla No. 2, Fig. 6), por décadas.
Tabla No. 1

EDAD:

I.U.E. en relación con la edad. 137 casos.

\begin{tabular}{|c|c|c|}
\hline EDAD (años) & No DE CASOS & $\%$ \\
\hline $20-29$ & 0 & 0 \\
\hline $30-39$ & 35 & $25.5 \%$ \\
\hline $40-49$ & 51 & $37.2 \%$ \\
\hline $50-59$ & 35 & $25.5 \%$ \\
\hline $60 \mathrm{y}$ mas & 16 & $11.7 \%$ \\
\hline Total & 137 & $100.0 \%$ \\
\hline
\end{tabular}

Figura No. 5

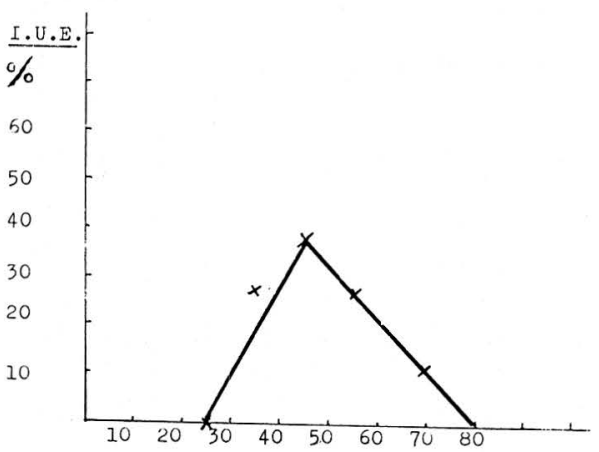

Tabla No. 2

EDAD:

Vejiga inestable con relación a la edad, 27 casos (16.4\%)

\begin{tabular}{|c|c|c|}
\hline $\operatorname{EDAD}($ años & No DE CASCS & $\%$ \\
\hline $20-29$ & 2 & $7.4 \%$ \\
\hline $30-39$ & 6 & $22.2 \%$ \\
\hline $40-49$ & 11 & $40.7 \%$ \\
\hline $50-59$ & 6 & $22.2 \%$ \\
\hline 60 y mas & 2 & $7.4 \%$ \\
\hline Total & 27 casos & \\
\hline
\end{tabular}


Figura No. 6

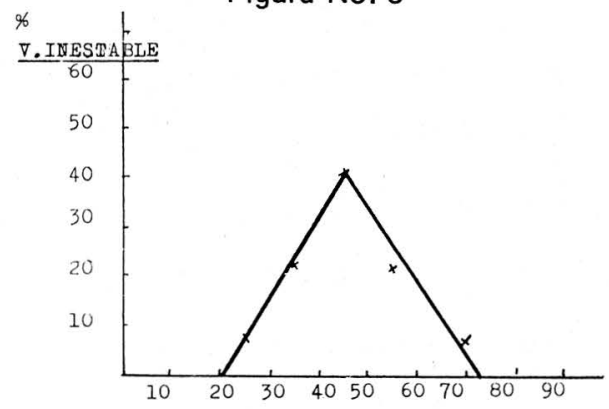

Paridad:

El número de hijos osciló entre nulíparas y un caso de 15 hijos, según se reporta por separado para I.U.E. (tabla No. 3, Fig. 7) y para V.I. (tabla No. 4 Fig. 8).;

Tabla No. 3

\section{PARIDAD:}

I.U.E. en relación con la paridad, 137 casos (83.5\%)

\begin{tabular}{|c|c|c|}
\hline RARTPAD S No & No de casos & $\%$ \\
\hline Nulipara & 0 & 0 \\
\hline $1-3$ & 25 & $18.2 \%$ \\
\hline $4-6$ & 53 & $38.6 \%$ \\
\hline 7 y mas & 59 & $43.0 \%$ \\
\hline Total & 137 & \\
\hline
\end{tabular}

Figura No. 7

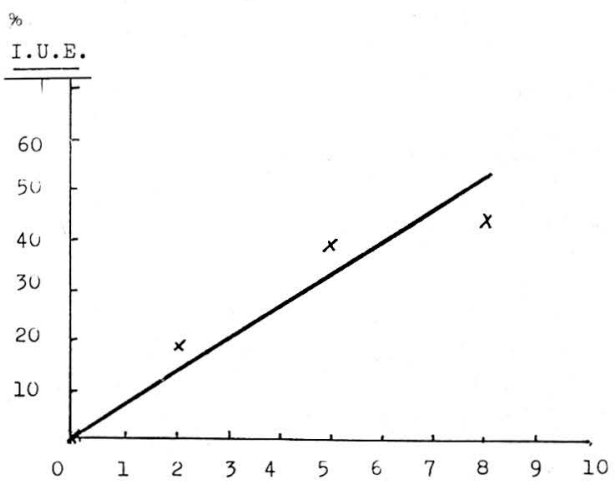

Tabla No. 4

PARIDAD:

Vejiga inestable en relación con la paridad, 27 casos (16.4응).

\begin{tabular}{|l|c|c|}
\hline $\begin{array}{c}\text { PARIDAD (NO } \\
\text { ie hijos) }\end{array}$ & No de casos & $\%$ \\
\hline Nulípara & 3 & $11.1 \%$ \\
\hline $1-3$ & 5 & $18.5 \%$ \\
\hline $4-6$ & 7 & $25.9 \%$ \\
\hline 7 y mas & 12 & $44.4 \%$ \\
\hline Total & 27 & \\
\hline
\end{tabular}

Figura No. 8

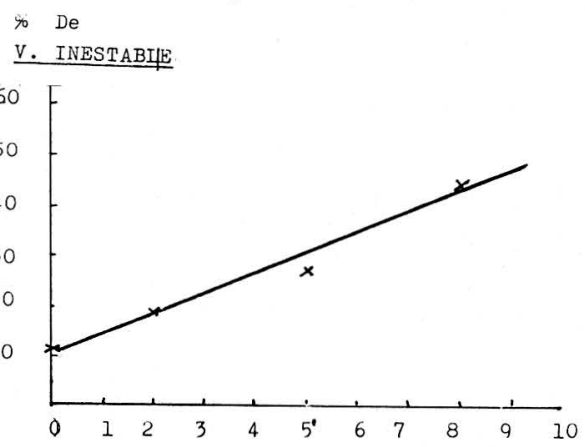

\section{Síntomas}

Condensamos en la tabla No. 5 los síntomas presentados por las 164 pacientes en órden de frecuencia.

\section{Antecedentes de trauma obstétrico}

Hubo 22 casos con antecedente de parto intervenido (13.4\%) de los cuales $13 \mathrm{co}$ rrespondieron a forceps (59) y 9 casos de espátulas de Velasco (41\%).

\section{Antecedentes médicos}

De los 164 casos estudiados encontramos 9 pacientes con Diabetes MeIlitus (5.5) de los cuales 6 presentaron V.I. (66\%). 
Tabla No. 5

\begin{tabular}{|l|c|c|}
\hline SINTOMA & No DE CASCS & $\%$ \\
\hline I.U. Grado II & 63 & $38.4 \%$ \\
\hline I.U. grado III & 101 & $61.5 \%$ \\
\hline I.U. en el coito & 96 & $58.5 \%$ \\
\hline I.U. postural & 86 & $52.4 \%$ \\
\hline Dispareunia & 86 & $52.4 \%$ \\
\hline Leucorreas & 85 & $52 \%$ \\
\hline Alguria & 60 & $36.5 \%$ \\
\hline Precuencia & 55 & $33.5 \%$ \\
\hline I. de Urgencia & 40 & $24.3 \%$ \\
\hline Enuresis & 21 & $12.8 \%$ \\
\hline $\begin{array}{l}\text { Palla control } \\
\text { voluntario }\end{array}$ & 10 & $12.8 \%$ \\
\hline
\end{tabular}

Seis de los 27 casos de V.I. son diabéticas, lo cual equivale al $22.2 \%$.

Antecedente de Cirugía para corregir I.U.

- Operación de Kelly 15 casos, de los cuales 5 casos de V.I.

- Operación de Tanagho 1 caso en el cual se confirmó V.I.

Total 16 casos, $9.7 \%$ del total.

\section{Síntomas}

Estos datos están condensados en la tabla No. 5 .

\section{Hallazgos de Cistoscopia}

Encontramos algunos hallazgos importantes en la uretrocistoscopia, los cuales evidencian la importancia de este procedimiento de rutina:
- Calibre uretral promedio ...... 22.5 FR

- Longitud uretral promedio ...... $3.6 \mathrm{~cm}$

- Capacidad vesical promedio . . . . . $475.5 \mathrm{~cm}$

- Menor capacidad vesical _.... $200 \mathrm{~cm}$

- Mayor capacidad vesical $\ldots \ldots .1 .000 \mathrm{~cm}$

- Uretras estrechas $\quad \ldots . .34$ casos (20.7\%)

- Traveculación vesical $\quad \ldots \ldots .7$ casos $(4.2 \%)$

- Pólipos intrauretrales $\quad \ldots . .44$ casos $(2.4 \%)$

- Divertículos intravesicales ..... 1 caso (0.6\%)

\section{Morbilidad}

La morbilidad subjetiva $y$ objetiva encontrada en los 137 casos quirúrgicos de mayor a menor frecuencia es como sigue:

- Dolor pélvico

24 casos (17.5\%)

- Alguria

21 casos (15.3\%)

- Frecuencia 14 casos (11.0\%)

- Infección urinaria

$10 \operatorname{casos}(7.2 \%)$

- Retención urinaria $8 \operatorname{cosos}(5.8 \%)$

- Recidiva de la I.U. $5 \operatorname{casos}(3.64 \%)$

- Hematomas de la herida $3 \operatorname{casos}(2.18 \%)$

- Absceso de pared 3 casos ( 2.18\%)

- Hipercorrección

1 caso $(0.7 \%)$

- Reintervención

\section{Detalle de los casos de recidiva}

$\begin{array}{rrrrr}\text { Grupo I } & 103 \text { casos } & \text { recidivaron } & 2 \text { casos } & 1.94 \% \\ \text { Grupo II } & 30 \text { casos } & \text { recidivaron } & 3 \text { casos } & 10 \% \\ \text { Grupo III } & 4 \text { casos } & \text { No hubo recidiva } & \\ \text { Grupo IV } & 27 \text { casos } & \text { recidivaron } & 6 \text { casos } & 22 \%\end{array}$

Recidiva combinada de los casos de I.U.E.: $\quad 3.64 \%$ (Sin incluir los casos de V.I.)

\section{COMENTARIOS}

1. La cirugía más frecuentemente practicada fue la operación de Tanagho, 103 casos, de los cuales en 10 casos (7.2\%) se aprovechó la misma vía abdominal para otro tipo de cirugía. 
2. De 30 casos de operación de Powell, en 13 (48\%) se aprovechó la misma vía para practicar otro tipo de cirugía diferente a la corrección de I.U. Además, la vía vaginal permite corregir mejor los casos de cistocele III.

3. Respecto de las pacientes con V.I., hablamos de mejoría y no de curación. Esta se evaluó subjetivamente mientras recibían el tratamiento médico. Es conocido que al suspenderlo las recaídas pueden presentarse hasta en el $50 \%$ de los casos (3). En este grupo es de esperar un porcentaje alto de deserción.

4. La I.U.E. aumenta progresivamente con la edad a partir de los 30 años, hasta la década entre los 40 a 49 años cuando alcanza su pico máximo y luego tiende a disminuir. Igualmente sucedió con los casos de V.I., pero se inició más temprano.

5. Tanto la I.U.E. como la V.I. aumentaron progresivamente con el número de hijos. No observamos casos de I.U.E. en nulíparas y si 3 casos de V.I.

6. Destacamos la diabetes como factor de riesgo para $\mathrm{V} . \mathrm{I}$.

7. La operación de Kelly fue el antecedente más frecuente de cirugía previa para corregir I.U., De estos hubo 5 casos de V.I., por lo cual se indica el estudio urodinámico cuando exista este antecedente.

8. Los criterios propuestos para solicitar urodinamia dan un porcentaje de acierto del $44.2 \%$ sin mayor riesgo de pasar desapercibida una V.I. cuando no se solicita.

9. Dos fracasos de 103 operaciones de Tanagho (1.94\%) lo consideramos satisfactorio si lo comparamos a otras series donde las recidivas oscilan entre el $7 \%$ y $10 \%(13,16,27)$. Todo parece indicar que la selección de las pacientes mejora el rendimiento.
10. El $10 \%$ de fracasos (3 casos) de la operación de Powell registrados, aparentemente alto, es susceptible de ser disminuido. Los tres casos se presentaron inicialmente cuando aún no estabamos familiarizados con la operación y se debieron a falla técnica.

11. En todos los casos de recaída esta se presentó antes de los dos meses, lo cual esta de acuerdo con la revisión de Lomanto y Gómez para la corrección de Kelly (14), pero es posible que la recidivas aumenten a 5 y 10 años.

12. De la operación de Stamey modificada de Pereyra, aún no podemos dar un concepto autorizado, dada nuestra baja casuística, por haber iniciado recientemente el grupo III, pero parece ser una estupenda al ternativa.

\section{CONCLUSIONES Y RECOMENDACIONES}

1. La I.U. en la mujer es un problema complejo que requiere estudio y manejo multidisciplinario. Es necesario clasificar el tipo de I.U. para excluir de tratamiento quirúrgico a las pacientes con inestabilidad vesical.

2. No es recomendable un sólo tipo de operación para corregir la I.U.E. Recomendamos acudir a varias técnicas como la de Tanagho, la de Powell o la de Stamey Pereyra. La escogencia se hace con criterio selectivo estudiando cada caso individualmente.

3. Recomendamos la operación de Kelly exclusivamente en los casos de I.U.E. grado I asociada a prolapso genital.

4. Un grupo para ampliar la futura investigación sería el formado por aqueIlas pacientes con I.U. mixta (I.U.E. más V.I.), en las cuales se plantea el tratamiento combinado médico y quirúrgico, con posibilidad de éxito. 


\section{CLINICAL TRIAL AND THERAPEUTIC APPROACH OF THE URINARY STRESS INCONTINENCE (USI) IN THE FEMALE}

\section{CONCLUSIONS AND RECOMMENDATIONS}

1. U.S.I. in the female is a complex problem that requires a multidisciplinary study and management. It is necessary to clasify the type of U.S.I. to exclude from surgical treatment patients with Vesical inestability.

2. It is not advisable an unique type of surgery for the correction of U.S.I. We reccomend to have recourse to various techniques as Tanagho, Powell or Stamey-Pereyra. The election is

\section{BIBLIOGRAFIA}

1. ALDRIDGE, A.H.: Transplantation of fascia for relief of urinary stress incontinence. Am Jou Obst \& Gyn, 44. 1942.

2. BURCH, J.C.: Urethrovaginal fixation to Cooper's ligament for correction of stress incontinence, cystocele and prolapse. Am. Jour Obst \& Gyn 81: 281, 1961.

3. DIAZ, J. HERNANDEZ, C.: Urodinamia en incontinencia urinaria femenina. Memorias primeras jornadas de Gin. y Obst. Clínica Fray Bartolomé de las Casas, Bog. 157, 1984.

4. ENHORNING, G.: Simultaneus recording of intravesical and intraurethral pressure in normal and stress incontinent: women. Acta chir svand, suppl 276, 1961.

5. ENHORNING, G.: Urethral closure studies with incoerent genography and simultaneus bladder urethra pressure recording Surg. Gyn. and Obst. 118: $507,1964$. done with a selective criterion studying individually each case.

3. We recommend Kelly operation for U.S.I. Grade I associated to genital prolapse.

4. A group for widening further investigation should be the conformed by those patients with a mixed type of U.S.I. (Urinary stress incontinence plus vesical inestability), in whom it is advised combined surgical and medical treatments with possibilities of success.

6. HODKINSON, C.P.: Stress urinary incontinence. Am. Jour. Obs. \& Gyn. 108: 1141, 1970.

7. GALLO, D.: Incontinencia urinaria por esfuerzo. Urología Ginecológica, primera edición, Guadalajara, 1979.

8. GREEN, T.H. Jr: The problem of urinary stress incontinence in the women. Obstet. and Gynecol. srv. 23: 603, 1968.

9. GREEN, T.H. Jr: Urinary stress incontinence: Diferential diagnosis, pathophysiology and management. American Journal Obstet. and Gynecology. 122: 368, 1975 .

10. GREEN, T.H. Jr.: Selección entre las vías de acceso vaginal o suprapúbica para el tratamiento quirúrgico de la incontinencia de alarma. Clin. Obs. y Gin. XII, 1977.

11. KASER, O.: Incontinencia de orina. Ginecología especial. Primera ed. Barcelona, cap. 7, 1977. 
12. KELLY, H.A.: Incontinence of urine in women. Urology and cutaneus review, 17, 291, 293, 1913.

13. LOMANTO, A. y cols.: Evaluación de la colpocistouretropexia en la incontinencia urinaria de esfuerzo. Rev. Col. Gin. y Ob. vol. XXXIII, 112, 1982 .

14. LOMANTO, A., GOMEZ, P.: Operación de Kelly. Rev. Col. Obst. y Gin. Vol. XXXIV, 28, 1983.

15. LOMANTO, A.: Incontinencia urinaria de esfuerzo en la mujer, anatomía, fisiopatología y diagnóstico. Memorias primeras jornadas de Gin. y Obs. Clin. Fray Bartolomé de las Casas, Bogotá, 91, 1984.

16. LOMANTO, A.: Incontinencia urinaria de esfuerzo en la mujer. Tratamiento. Memorias primeras jornadas de Gin. y Obs. Clínica Fray Bartolomé de las Casas, Bogotá, 111, 1984.

17. LOPEZ, A., CORZO, J.E.: Corrección de la Incontinencia urinaria de esfuerzo por medio de la uretrocistopexia retropúbica por vía vaginal. Rev. Col. Obst. y Gin. vol. XXXIV No. 34, 1983.

18. MARSHALL, V.F.: MARCHETTI, A.A., KRANTZ, K.E.: The correction of stress incontinence by simple vesicourethral suspension. Surgery, Gynecology and Obstetrics, 88, 509, 518, 1949.

19. PEREYRA, A.J. y cols: Combined urethrovesical suspensión and vaginourethroplasty of stress incontinence. Obs. Gyn. 1967.
20. PACEY, K.: Pathology and repair of genital prolapse. J. Obs. \& Gyn. of the Brit, 56, 1949.

21. Powell, L.C. Jr.: Retropubic uretrocystopexy: Vaginal approach. Am. J. Obstet. and Gyn. 140: 91, 1981.

22. REDONDO, H., LOMANTO, A.: Incontinencia urinaria de esfuerzo: Revisión de la operación de Kelly en el Instituto Materno Infantil de Bogotá, trabajo no publicado, 1980.

23. STAMEY, T.A.: Surgery of female incontinence. Am. Jour. Obst. and Gyn. 77, 1980.

24. TANAGHO, E.A.: Observations on the dinamics on the bladder neck. Brit. Jour. Urol, 38; 72, 1966.

25. TANAGHO, E.A., and cols.: Mechanism and urinary continence. Embriogic, anatomic and pathologyc considerations. Brit. Jour. Urol, 100: 640, 1968.

26. TANAGHO, E.A. and cols: The trigone: Anatomical and physiologycal considerations in relation to the urethrovesical junction. J. Urol Am 100: 623, 1968.

27. TANAGHO, E.A. and cols: Colpocistouretropexia: La vía; como la hacemos. J.; Urol, vol. 116. 1974.

28. TANAGHO, E.A. and cols: Colpocystourethropexy the way we do it. J. Urol, 110751, 1976.

29. ULLERY, J.C.: Treatment of stress incontinence in female. Am. J. Obst. \& Gyn. 66: 853, 1953. 